\title{
PREDICTING FUTURE BEHAVIOR OF TRANSIENT EVENTS RAPIDLY ENOUGH TO EVALUATE REMEDIAL CONTROL OPTIONS IN REAL-TIME
}

Steven Rovnyak*
Student Member

Chih-Wen Liu*

Student Member

* Cornell University

Ithaca, NY 14850
Jin Lu**
Member

**

\author{
Weimin $\mathrm{Ma}^{*}$ \\ Member
}

James Thorp*

Fellow
Keywords: Clustering, estimation, integration, pattern recognition, phasor measurements, transient stability.

ABSTRACT - Electric utilities are becoming increasingly interested in using synchronized phasor measurements from around the system to enhance their protection and remedial action control strategies. Accordingly the task of predicting future behavior of the power system before it actually occurs has become an important area of research. This paper presents and analyses several approaches for solving the real-time prediction problem. The first method clusters the initial post-fault swing curves into coherent groups and fits a low order equivalent model to the specific transient event in progress. The model is updated with each new set of phasor measurements and provides a running prediction of future behavior which is valid for approximately $1 / 2$ second into the future. We show how this capability would be useful inside the framework of a protection scheme such as the proposed French Defence Plan.

If on the other hand a relatively detailed rectuced-order model is available ahead of time, then it could be used to predict future behavior for several different control options. The task in this case is to solve the model much faster than real-time using the post-fault phasor measurements as the initial condition. In order to solve systems with detailed load models fast enough for real-time prediction, we present a new piecewise constant current load model approximation technique that can solve a model as complex as the New England 39 bus system with composite voltage dependent loads much faster than real-time. If the reduced order model is too large for real-time solution, then a pattem recognition tool such as decision trees can be trained off line to associate the post-fault phasor measurements with the outcome of future behavior. In this case also, the piecewise constant current technique would be needed to perform the off-line training set generation with sufficient speed and accuracy.

\section{INTRODUCTION}

Synchronized phasor measurement units (PMU's) simultaneously measure state variables in remote locations of the power system network [1]. The phasors obtained from a period or more of samples from all three phases provide a precise estimate of the positive sequence voltage phasor at each installation. Commercially available systems based on Global Positioning System (GPS) satellite time transmissions can provide synchronization to 1 microsecond accuracy, which means that relative phase angles can be measured to a precision of 0.02 electrical degrees [2]. Utility experience indicates that

\footnotetext{
94 SM 518-1 PWRS A paper recommended and approved by the IEEE Power System Engineering Committee of the by the IEE Power System Society for presentation at the IEEE/PES 1994 Summer Meeting, San Francisco, CA, the IE $24-28,1994$. Manuscript submitted January 4, 1994 ; made available for printing May 3, 1994.
}

communication systems can transmit these time-tagged phasor measurements to a central location every 5 cycles [3]. It is therefore possible to track the relative phase angles of important state variables in real-time.

An emerging application of this technology is to track the state of the system immediately following a transient event in order to select an appropriate remedial control action. One such real-time control strategy is already being implemented at the Florida-Georgia interface [4], and others are currently under development [5]. This research was performed under a subcontract of the Florida-Georgia project, which was sponsored by EPRI and installed at the interface between the two regions. An important feature of the Florida-Georgia situation is that inter-area oscillations between the two regions can always be modeled as a two-machine equivalent system. When such oscillations are initiated, phasor measurements are taken within Florida and Georgia in order to infer the corresponding state of the two-machine equivalent. Future stability is then determined by applying the equal area criterion. This prediction is used for adaptive out-of-step relaying at the Florida-Georgia interface.

\section{REAL-TIME PREDICTION}

Our research addressed the question of accomplishing out-of-step prediction when the system does not always reduce to a previously known two-machine equivalent. Possible methods of approaching this problem which we have researched fall into two broad categories:

(1) Infer a small-size (e.g. 2, 3 or 4 machine) equivalent from the post-fault phasor measurements, which models the particular mode of oscillation of the fault in progress. Solve the model forward in time in order to predict future behavior.

(2) Use a reduced-order but relatively detailed model of the system (e.g. the 39 bus model for New England) which adequately covers the many modes of oscillation initiated by different contingencies. Solve the model faster than real time if computational resources permit, or else train a pattern recognition tool off-line in order to associate in real-time the post-fault phasor measurements with the outcome of future behavior.

\subsection{Clustering-Estimation-Integration (CEI)}

The first strategy is accomplished, in a limited fashion, without any prior knowledge about the system on which it is performed. Section 3 in this paper presents a method for deducing in real-time which machines are swinging together, and estimating the parameters of a 2,3 or 4 machine equivalent which is then solved faster than real-time. This technique would be useful inside the framework of the proposed French Defence Plan which will utilize phasor measurements to guard against losses of synchronism $[5,6]$. The objective of this plan is to implement a controlled separation of the system into "islandable" areas whenever a loss of synchronism is detected by the PMU's. An issue of critical importance in this scheme is the amount of time between the detection of phase angle opposition and the implementation of islanding. Given the technological constraints, 
a time delay of 1.3 seconds or less has been chosen to be acceptable. This time scale, it should be noted, is much faster than the operation of standard under-frequency relays. In a panel discussion on phasor measurement applications at the 1993 PES Summer Meeting, a representative of Electricité de France [7] mentioned the difficulty in reacting quickly enough to the detection of loss of synchronism, and indicated the desirability of predicting future behavior. Accordingly, we show that the proposed technique can reliably predict losses of synchronism a short time into the future.

As illustrated in Section 3, our clustering - estimation integration (CEI) technique can be used to provide a continually updated prediction window extending approximately half a second into the future. Instead of waiting for physical loss of synchronism to occur, it would be possible to act in advance on the basis of future predicted behavior. We simulate the capability of the CEI prediction technique in giving advance waming of loss of synchronism and show that it can predict with some accuracy which generators will go over and under-speed. This is enough information to implement the convrolled separation ahead of time. The performance is not perfect, but the errors that do occur tend to be tolerable. For example, if a subset of the over-speed generators are separating faster than the rest then the algorithm will predict at first that only these will go over-speed. However such errors could be accommodated by resuming prediction for the remaining machines. And furthermore, if the system can only be islanded in a limited number of ways, then it would still make sense to separate the areas containing the most rapidly diverging machines. Another source of error is that the length of advance warning before loss of synchronism is not uniform, and occasionally there is no warning. As a consequence the CEI glgorithm must be viewed as a potential augmentation to a scheme such as the Defence Plan, which will improve reaction times in many cases, and will cause little or no harm in others.

\subsection{Detailed Reduced-Order Model}

The second approach listed above would be required for predicting future behavior under different control options, because the first approach extrapolates the future behavior entirely from past observations. If one had a reduced-order model in advance of the contingency then one could use the most recent phasor measurements as the initial condition, and simulate what would happen for different control actions. We have not yet addressed the derivation of this model, but we have covered the possibilities for its solution. If the model is small enough, then it can actually be integrated much faster than real time in order to predict future behavior before it occurs. Section 4 in this paper demonstrates that a model as detailed as the New England 39 bus system with composite voltage dependent loads can be solved much faster than real time using modern workstation computers. In that section we present an efficient piecewise constant current load model approximation technique, which is conceptually similar to the Dynamic Ward-Type Equivalent model $[8,9]$, but addresses unresolved assumptions about the updating of coefficient matrices.

If the reduced-order equivalent model is too complex for real-time solution, then a pattem recognition technique may be employed. In [10] decision trees are trained to predict the future system stability from a short sampling of the post-fault phasor measurements. Using the New England 39 bus test system for this experiment, we find that decision trees achieve very high accuracy $(>95 \%)$ while demonstrating robustness to measurement imprecision, as well as small random variations in the operating point. The decision tree building process can be completely automated, and a new tree can be constructed for this system in just a few minutes. This feature is essential because changing system conditions will require frequent updates of the decision logic. Fortunately, the training set for each new tree can be generated in parallel, and this was done on a cluster of five IBM RISC System $/ 6000$ computers. Using the parallel RS/6000's, training set generation for the 39 bus system takes 2-3 minutes, and subsequent tree-building from this data requires 62 seconds on a single RS/6000 computer. Because the potential payoff from system-wide instability detection will arguably permit these computational requirements to be met, the pattem recognition approach allows greater flexibility in selecting the model used to generate the training data.

Computational resources are still an important ooncern in the pattem recognition approach, however, and having an efficient solution method will enable more detailed simulations to be run. Some power system models are well known for giving optimistic results - predicting stability in the case of instability, while others give conservative results. The constant impedance load model generally gives optimistic results, while the constant P-Q load model generally gives conservative results [11]. It has been shown that better generator and load models give more accurate results $[12,13]$. Since a decision tree classifier learns to predict future behavior on the basis of simulated data, it is important to use a reasonably accurate model. The decision tree paper [10] uses the constant impedance model, and so there exists both a need and an opportunity to explore the efficacy of this approach using models of greater complexity. The piecewise constant current load model approximation technique presented in Section 4 will enable the rapid solution of composite load model power systems, which will improve training set accuracy for the pattem recognition approach.

The issue of deriving a reasonably accurate reduced-order model is somewhat new due to the trade-off between speed and accuracy required by the real-time prediction problem. The question of deriving a highly accurate reduced-order model has already been addressed in the context of planning studies, where a 50\% reduction in simulation time constitutes significant savings $[14,15]$. Real-time prediction, however, requires a substantially reduced-order model in order to solve it in real-time or else train a decision tree from a large number of off-line simulations. As was shown in the Florida-Georgia application [4], even a two machine model can potentially give much better results than trying to predict losses of synchronism using only local measurements. We therefore postulate that a model of moderate complexity such as the 39 bus system for New England [16], or the 131 bus system for the western United States [17] can potentially predict future behavior with sufficient accuracy to evaluate the effects of candidate remedial control options such as controlled separation or DC line power flow modulation. Unfortunately most of these reduced models were developed for the purpose of creating a reasonably sized test system, rather than attempting to strictly replicate the behavior of the larger system. Some of them do nevertheless replicate the major modes of oscillation relatively well. It is clear at least that some reduction can be performed while maintaining reasonable accuracy, and so we address the real-time solution for reduced-order models of all different sizes. Specifically, we show in Section 4 that models up to the size of the 39 bus system with composite nonlinear loads can actually be solved in real-time, whereas larger models would require using the pattern recognition approach. The same piecewise constant current load approximation technique, however, would prove valuable in performing the off-line simulations needed for the pattem recognition approach as well.

Section 3 below presents the clustering - estimation integration (CEI) technique, described earlier in Section 2.1, which extrapolates the dynamic behavior a short time into the funure without using any prior knowledge of the power system model. Section 4 then presents the piecewise constant current load model approximation technique which can rapidly solve a moderately complex, reduced-order model with composite nonlinear loads.

\section{CLUSTERING-ESTIMATION- INTEGRATION (CEI) ALGORITHM}

The method described in this section fits a 2,3 or 4 machine equivalent model to the swing data observed by the PMU's, and uses that model to roughly predict furure behavior before it 
actually occurs. Like the Extended Equal Area Criterion [18], this method attempts to model the mode of oscillation of a particular disturbance with a very low-order equivalent system. An important difference, however, is that our method only solves the model $1 / 2$ to 1 second forward, rather than attempting to predict the ultimate stability or instability. While this information would not be very useful in planning studies, it can provide crucial lead time between the prediction of disaster and the occurrence of disaster in the context of the adaptive out-of-step protection problem.

This approach relies on the fact that a small sized equivalent system can actually be integrated much faster than real-time. For example the system trajectories for a 4 machine system can be integrated one second into the future in just 0.01 seconds of CPU time on a Sun SPARC Station IPX computer. The reduced-order model is derived by observing the post-fault swing curve data for a fraction of a second, and clustering the swing curves into coherent groups. The centers of angle of the identified groups are computed, and the corresponding reduced-order equations are discretized in order to perform least-squares parameter estimation of the system coefficients from the observed swing curve data. The equivalent system is integrated using the most recent set of phasor measurements as the initial condition, and the results are consistently accurate for approximately $1 / 2$ second into the future. By predicting the future behavior of the centers of angle of the coherent groups, it is possible to determine in most cases which machines will go over and under speed. Our simulations show that using a 2 machine equivalent has several advantages, such as requiring the least amount of data for estimating the model parameters, and producing tolerable errors when predicting future behavior.

\subsection{Clustering and Parameter Estimation}

Of the many clustering algorithms [19], joining and agglomeration (i.e. aggregation) seems particularly suited to the real-time transient stability problem. If you wish to cluster $N$ objects into $m$ groups, and you can combine any two objects into one, the joining and agglomeration algorithm says to combine the two closest objects and repeat until $m$ objects remain. The center of angle (COA) computation provides a natural method for combining any two swing curves. The total distance between two curves is computed as the squared distance summed over the length of the observation window after the swing curves are shifted to start at zero. Hence this distance measure roughly calculates the squared $L_{2}$ distance between two curves over the observation window, after the curves have been located with a common starting point. Machines which swing together will have small computed distances between them and diverging machines will accrue larger distances as the observation window lengthens. It should be pointed out that the center of angle computation requires a unit of mass to be associated with each of the swing curves observed by the PMU's, and in this sense requires a rudimentary knowledge of the power system dynamics.

Our simulations in Section 3.2 will show that 3 and 4 machine equivalent systems require too many observation samples and/or unrealistic precision in the measurements. For this reason we only describe the estimation process for the 2 machine equivalent. Generalization to multiple machines is straightforward except for the question of reducing the number of equations by coordinate transformation (e.g. center of angle (COA), or taking one machine as the reference). Whereas the 2 machine equivalent reduces neatly to one equation by coordinate transformation, reducing larger systems produces more terms per equation and is therefore counterproductive in this situation.

The swing equation for a two machine equivalent has 3 coefficients $\mathrm{P}, \mathrm{A}$ and $\mathrm{B}$ :

$$
\delta=\mathrm{P}+\mathrm{A} \cos (\delta)+\mathrm{B} \sin (\delta)
$$

Phasor measurement samples from the observation window are used to calculate the COA's of the equivalent machines identified by the clustering algorithm. In the 2 machine equivalent $\delta(k)$ represents the difference between the COA's at sample time $k$, and will be used to estimate the coefficients $P, A$ and $B$. The second derivative can be approximated as:

$$
\delta(k)=\frac{\delta(k+1)-2 \delta(k)+\delta(k-1)}{(\Delta t)^{2}}
$$

Where $\Delta t$ is the amount of time between samples. Substituting these values of $\delta(k)$ back into the swing equation produces a set of equations which are linear in $\mathrm{P}, \mathrm{A}$ and $\mathrm{B}$ :

$$
\left[\begin{array}{lll}
1 & \cos \delta(2) & \sin \delta(2) \\
1 & \cos \delta(3) & \sin \delta(3) \\
1 & \cos \delta(4) & \sin \delta(4)
\end{array}\right]\left[\begin{array}{l}
\mathrm{P} \\
\mathrm{A} \\
\mathrm{B}
\end{array}\right]=\frac{1}{(\Delta \mathrm{t})^{2}}\left[\begin{array}{l}
\delta(3)-2 \delta(2)+\delta(1) \\
\delta(4)-2 \delta(3)+\delta(2) \\
\delta(5)-2 \delta(4)+\delta(3)
\end{array}\right]
$$

In general the number of equations will be two less than the number of samples in the observation window. Assuming that phasor measurements are collected every 4 cycles, the minimum observation window length for a two machine equivalent is 20 cycles $(1 / 3$ second), i.e. 5 sample points. When the linear equations are over specified (more equations than parameters) then the least-squares estimation technique can be used. We utilized the Engineering and Scientific Subroutine Library (ESSL) to perform this estimation.

\subsection{Simulation Results}

We tested the performance of the CEI prediction technique on the New England 39 bus test system under two different loading conditions. The rationale for using this system is that it roughly represents having 10 well placed PMU's embedded in a larger system. The 39 bus model presents a wide variety of oscillatory modes, some of which are difficult to capture with a reduced-order equivalent. On the other hand there are plenty of well-behaved cases for which the method works perfectly. It was only through extensive simulation that we were able to document the shortcomings of the CEI method using 3 and 4 machine equivalents. This experience serves to illustrate the fact that it is generally not sufficient to demonstrate the success of a particular method on just a few examples, rather that broader testing is required. We were fortunate to have access to the Comell National Supercomputer Facility, which allowed us to quickly run many thousands of simulations during the development of the algorithm.

The testing methodology used here is consistent with previous work [10] and assumes collecting samples from each of the 10 generators every four cycles. These simulated measurements are tuncated to 0.001 radians of precision before estimating the parameters of a reduced-order system. Three phase short-circuit to ground faults with random locations and durations are simulated on each of the 34 transmission lines. The faulted line is always removed at clearing time. Choosing 30 disturbances at random for each transmission line produces a test set of 1020 cases. Since this method does not require prior knowledge about the power system dynamics, we create two test sets from substantially different operating points. The first uses the New England data directly from $P$ ai [16], and the second has $25 \%$ increased real power loading as in [10]. Faults from 1-12 cycles in duration were simulated for the low load case and faults from 1-8 cycles were simulated on the high load case. Because the high load case is more susceptible to instability, there are more unstable contingencies in the high load test set even though the fault durations are shorter.

We simulate the calculation of a "running prediction" which is first made after observing a pre-specified number of samples and then updated with each new sample. Every case in the test set contains 60 samples of the phase angle measurements spaced 4 cycles apart for a total of 4 seconds of post-fault swing curve 
data. The running prediction continues until the observation window reaches 45 samples ( 3 seconds) in length. Depending on the amount of time beyond the most recent measurement sample for which prediction is attempted, the short-term outlook from a given prediction calculation will indicate either future stability or instability. When during the course of a disturbance loss of synchronism is never predicted, then no action should be taken. When the prediction of instability occurs for the first time, the associated control logic may either act immediately or wait for additional evidence from subsequent predictions. In simulation we found that predicting stability or instability 6 samples ( 24 cycles) ahead of time without double-checking gives consistent results.

For each case in the test set, it is determined whether the running prediction ever declares instability while following a stable swing. Such a false alarm would cause unnecessary system islanding and should be avoided. For the unstable swings it is determined whether the method predicts instability ahead of time, and records the amount of lead time between the prediction of instability and the actual loss of synchronism. The earliest that one could detect loss of synchronism without the aid of prediction is when any two machines have reached a phase angle difference of 180 degrees. In practice, additional time could be required to determine which of the many machines are going over and under-speed. Hence for practical purposes, the advance waming may actually be longer than the times given here.

The criteria of false alarms and advance waming times can be used to point out the deficiencies of the 3 and 4 machine equivalents for the CEI method. A three machine equivalent has 5 parameters per equation and a four machine equivalent has 7 parameters per equation. Therefore a minimum of 7 and 9 data samples respectively are needed to estimate the coefficients of the 3 and 4 machine equivalents. Our simulations used 8 and 10 samples respectively in order to decrease problems with accuracy. Nevertheless, the three machine equivalent has a false alarm rate of $22 \%$ and the four machine equivalent has a false alarm rate of $61 \%$ using the data set from the nominally loaded operating point.

Having obtained such poor results for the 3 and 4 machine equivalents we needed to verify the operation of the CEI estimation program. This was easily checked by repeating the above tests with higher precision data. Instead of truncating the simulated phasor measurements to three decimal places of precision, we tried using five decimal places of representation. For the same data set, and beginning the running prediction at the earliest possible moment (after 7 and 9 samples respectively for 3 and 4 machines), the false alarm rates decreased to $1.7 \%$ and $5.8 \%$ respectively. Since five decimal places of precision is completely unreasonable for PMU's, we wondered whether using more samples of the lower precision data could achieve the same effect. Indeed, using 12 and 16 samples respectively also reduces the false alarm rates to $1.1 \%$ and $8.1 \%$ for the 3 and 4 machine equivalents. However the time delay between fault clearing and the first prediction is so long ( 12 samples $=0.8$ seconds) that only $26 \%$ and $16 \%$ respectively of the unstable cases are predicted with any advance warning for the 3 and 4 machine equivalents. We therefore conclude that the 3 and 4 machine equivalents require more information for parameter estimation than is available from a short window of realistic-precision phasor measurement data.

The 2 machine equivalent for the CEI method performs well on all accounts. If the running prediction is allowed to begin after the first five samples of post-fault data, the method has a $1.8 \%$ false alarm rate for the nominally loaded system and a $2.2 \%$ false alarm rate after the loading is increased 25 percent Experimenting with higher precision data and longer observation windows, we find that false alams can be completely eliminated by using five decimal places of precision or else by lengthening the minimum observation period to 8 samples ( 32 cycles). Given that the higher precision is unattainable, one must choose between faster response times and fewer errors. Our simulations show that in order to predict most of the unstable cases in advance, that the shorter minimum observation window is necessary. For this reason we will discuss the results for the 2 machine equivalent with prediction starting at 5 samples $(0.33$ second) after the fault clearing time.

Table I: Unstable Cases by Length of Advance Warning Advance Warning in Cycles $(1 / 60 \mathrm{sec})$

\begin{tabular}{llllllllll}
\hline 0 & 4 & 8 & 12 & 16 & 20 & 24 & 28 & 32 & $36+$
\end{tabular}

$\begin{array}{lllllllllll}\text { \# cases (*) } & 47 & 31 & 31 & 28 & 21 & 29 & 3 & 0 & 0 & 0\end{array}$

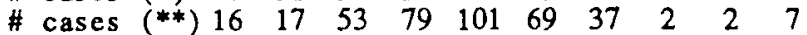

${ }^{*}$ ) From nominal loading test set

(**) From $25 \%$ increased loading test set

Table I shows the amount of advance waming between the prediction of instability and the actual occurrence of 180 degree phase angle difference between any two generators. The first occurrence of 180 degree phase angle difference between PMU locations is the earliest that one could possibly initiate remedial control in the absence of prediction. Therefore the time gained by predicting instability, if action could be taken on its basis, would arguably be longer than given in Table $I$. Nevertheless, because of the time constraints on taking remedial control, even $1 / 4$ second ( 15 cycles) of early warning is significant in this application. Sometimes the technique does not give any waming of instability, and these cases constitute "false dismissals". In the proposed framework, false dismissals will produce the same result as if there were no prediction algorithm in place because no remedial control action will be taken in either case.

One must predict the specifics of future behavior in order to select an appropriate remedial control action in advance. Within the French Defence Plan, for example, one needs to know how the generator phase angles are going to separate in order to prescribe the correct pattern of islanding. In simulation we found that the diverging generators could be predicted with reasonable accuracy by integrating the reduced-order system 1.3 seconds forward from the observation at which instability was first detected, and comparing this with the true system generator angles 1 second past the onset of 180 degree phase difference. The reason for integrating 1.3 seconds in the reduced system is that prediction of instability typically occurs approximately 0.3 seconds before the onset of 180 degree phase difference in the full system. If any equivalent machine has deviated from the center of angle of the reduced system by $\pm \pi$, then the generators corresponding to that equivalent are predicted to go over or under speed respectively. The strict criterion for whether this prediction is valid is whether the prediction for each generator matches the actual behavior in the full system.

Our simulations show that in most cases where instability is predicted in advance, that the details are correct according to the above criterion. Of 190 unstable cases from the nominal loading test set, 47 occur without any advance warning. In these 47 cases, controlled separation will be determined by the actual observation of pole slip rather than prediction, so the accuracy of prediction in these cases is not pertinent. Of the remaining cases, 117 are predicted correctly in detail whereas 26 are not. For the 25 percent increased loading situation, only 16 of the unstable cases are false dismissals. Of the remaining 367 unstable cases, 275 are predicted correctly in detail and 92 are not. In those cases where the details are incorrect, it is necessary to examine the nature and severity of the errors.

Fortunately the misclassifications of unstable cases tend to be tolerable when using the 2 machine equivalent. Most of these result from clustering the initially fastest machines into their own separate group. Examining the 118 misclassified cases from both test sets, we find that 108 represent the situation where the initially fastest machines are predicted to go over speed, while the rest are predicted to remain within $\pm \pi$ of the center of angle. As a consequence only a subset of the diverging generators are predicted to go over speed. We have already 
suggested that it would be reasonable to separate the area containing the initially fastest machines, and resume prediction for the others. Of the 10 remaining cases, 4 predict instability without specifying which machine angles will diverge from the rest. Since no control action would be taken, these cases are essentially false dismissals.

Three cases predict that a subset of the accelerating generators will deviate in the positive direction while the rest diverge in the negative direction. Presumably the control response would be to separate the one set from the other, and it would soon become apparent that further separation was necessary. Finally, the 3 remaining cases predict that both groups diverge from the center of angle whereas the detailed simulation shows that only one group will deviate from the center of angle by more than $\pi$ within the first second after instability. However both groups will have already separated by more than 180 degrees from each other, and both will ultimately deviate from the center of angle by more than $\pi$. Hence the separation based on the prediction would be appropriate to the actual behavior.

This concludes our discussion of the proposed CEI method for predicting future behavior in the absence of a previously known reduced-order equivalent. Since the method extrapolates future behavior on the basis of past observations, it cannot predict what would happen under different control options. There is reason to believe, however, that a reduced-order equivalent model can be obtained in advance having sufficient accuracy to provide useful assessment of candidate remedial control options. Since there is no guarantee that such a model can be solved faster than real-time, we propose using a pattem recognition methodology as a backup approach $[10,20]$. In either case, it is necessary to have an efficient and accurate method for solving the power system behavior. Accordingly we propose the following piecewise constant current load model approximation technique for power systems having composite voltage dependent load models.

\section{PIECEWISE CONSTANT CURRENT}

The idea behind the piecewise constant current approximation technique is to begin with a model accurate for the initial point of the trajectory and then replace the model each time the trajectory leaves the area where the linearization is valid. Each new model is linearized about the most recent trajectory point. The criterion for re-computing the new model is whether any of the generator angles have become farther than $\gamma$ from the current linearization point. Values of $\gamma$ on the order of 5 to 10 degrees give good simulation results relative to the exact solution method for constant PQ loads.

Our method is conceptually similar to the Dynamic Ward-Type Equivalent solution technique $[8,9]$. Both achieve computational efficiency for solving power system dynamics involving voltage dependent and constant $P Q$ loads by making piecewise linear approximations to the load flow equation nonlinearities, which are updated as necessary in order to preserve the validity of the linearization. In investigating the speed of computation for the Ward-Type Equivalent, we assumed that the coefficient matrices for each successive linearization could be pre-computed and stored in memory for use during the transient stability computation. The question of whether those computations could be performed ahead of time remains an unresolved issue, and it is worthwhile to note that the number of linearization regions which could be traversed by the set of all possible post-fault swing trajectories is potentially very large. This concem motivated us to produce an analogue to the Dynamic Ward-Type Equivalent algorithm which even executes quickly while updating the linearization in real-time. The piecewise constant current technique described in this section uses the fast decoupled power flow method to perform the loadflow calculation needed to update the linearization. The timing results given at the end of this section include the computation of the successive linearizations encountered during the course of the post-fault swing trajectory.
The remainder of this section describes the piecewise constant current technique and outlines the generalization to composite nonlinear load models. We first assume constant PQ loads. A loadflow computation must be performed whenever re-calculating the linear model, because the quantities $\mathrm{V}_{\text {load }}, \mathrm{Q}_{\mathrm{gen}}$, and $\delta$ must be derived from $V_{\text {gen }}$. Q Q oad, and $P$. Consider the loadflow equations given $(P, Q)=\left(P_{0}, Q_{0}\right)$ where $\left(P_{0}, Q_{0}\right)$ represent constant $\mathrm{PQ}$ loads. Looking at the i'th loadflow equation, we have

where

$$
\begin{aligned}
& 0=\mathrm{P}_{\mathrm{Oi}}+\mathrm{g}_{\mathrm{i}}(\delta, \mathrm{V}) \\
& 0=\mathrm{Q}_{0 \mathrm{i}}+\mathrm{h}_{\mathrm{i}}(\delta, \mathrm{V})
\end{aligned}
$$

$$
\begin{aligned}
& \mathrm{g}_{\mathrm{i}}(\delta, \mathrm{V})=\sum_{J} \mathrm{G}_{\mathrm{ij}} \mathrm{V}_{\mathrm{i}} \mathrm{V}_{\mathrm{j}} \cos \left(\delta_{\mathrm{i}}-\delta_{\mathrm{j}}\right)+\sum_{j} \mathrm{~B}_{\mathrm{ij}} \mathrm{V}_{\mathrm{i}} \mathrm{V}_{\mathrm{j}} \sin \left(\delta_{\mathrm{i}}-\delta_{\mathrm{j}}\right) \\
& \mathrm{h}_{\mathrm{i}}(\delta, \mathrm{V})=\sum_{j} \mathrm{G}_{\mathrm{ij}} \mathrm{V}_{\mathrm{i}} \mathrm{V}_{\mathrm{j}} \sin \left(\delta_{\mathrm{i}}-\delta_{\mathrm{j}}\right)-\sum_{j} \mathrm{~B}_{\mathrm{ij}} \mathrm{V}_{\mathrm{i}} \mathrm{V}_{\mathrm{j}} \cos \left(\delta_{\mathrm{i}}-\delta_{\mathrm{j}}\right)
\end{aligned}
$$

Specifying $P$ and $Q$ values at the load buses and $P$ and $V$ values at the generator buses, the loadflow equations can be solved for $\mathrm{V}$ and $\delta$ at the load buses and $\mathrm{Q}$ and $\delta$ at the generator buses.

In the more general case we will have composite loads:

$$
\begin{aligned}
& \mathrm{P}_{\text {load }}=\mathrm{P}_{0}-\mathrm{I}_{0} \mathrm{~V} \cos \left(\delta-\varphi_{0}\right)+\left[\mathrm{V}^{2} / \mathrm{Z}\right] \cos \theta+\mathrm{P}_{\mathrm{vd}}(\mathrm{V}) \\
& \mathrm{Q}_{\text {load }}=\mathrm{Q}_{0}-\mathrm{I}_{0} \mathrm{~V} \sin \left(\delta-\varphi_{0}\right)+\left[\mathrm{V}^{2} / \mathrm{Z}\right] \sin \theta+\mathrm{Q}_{\mathrm{vd}}(\mathrm{V})
\end{aligned}
$$

where $\left(\mathrm{P}_{0}, \mathrm{Q}_{0}\right)$ is the constant $\mathrm{PQ}$ portion of the particular load, and $\mathrm{L}_{0} \angle \varphi_{0}$ is the portion of load represented as a constant current source flowing into the network such that the angle $\varphi_{0}$ is on the same reference as $\delta . Z \angle \theta$ represents the constant impedance part, and the functions $P_{v d}$ and $Q_{v d}$ are other voltage dependent parts. Note that the load equations can be rewritten in the form:

$$
\begin{aligned}
\mathrm{P}_{\text {lowd }} & =\mathrm{P}_{0}+\mathrm{P}_{\mathrm{nl}}(\delta, \mathrm{V}) \\
\mathrm{Q}_{\text {load }} & =\mathrm{Q}_{0}+\mathrm{Q}_{\mathrm{nl}}(\delta, \mathrm{V})
\end{aligned}
$$

where $P_{n l}$ and $Q_{n l}$ are known functions of $V$. Letting

$$
\begin{aligned}
& \hat{\mathrm{g}}_{\mathrm{i}}(\delta, \mathrm{V})=\mathrm{P}_{\mathrm{nl}_{i}}(\delta, \mathrm{V})+\mathrm{g}_{\mathrm{i}}(\delta, \mathrm{V}) \\
& \mathrm{h}_{\mathrm{i}}(\delta, \mathrm{V})=\mathrm{Q}_{\mathrm{nl}_{i}}(\delta, \mathrm{V})+\mathrm{h}_{\mathrm{i}}(\delta, \mathrm{V})
\end{aligned}
$$

we see that the power flow equations for composite loads have the same form as the corresponding equations for constant PQ loads. Therefore the piecewise technique, which relies on a loadflow calculation to update the equivalent parameters, can handle composite voltage dependant loads in the same manner as constant PO loads. Bearing this in mind, the following section applies the piecewise constant current technique to a system with constant PQ loads.

\subsection{Piecewise Constant Current Approximation}

After performing a loadflow as indicated above, we compute the equivalent current injection $\mathrm{I}_{1 j}<\varphi_{i_{j}}$ corresponding to the values of $P_{j}, Q_{j}$ at each load bus $j$

$$
I_{\mathrm{lj}}<\varphi_{\mathrm{lj}}=\left(\mathrm{P}_{\mathrm{j}}^{2}+\mathrm{Q}_{\mathrm{j}}^{2}\right)^{1 / 2} / \mathrm{V}_{\mathrm{lj}}<\left(\delta_{\mathrm{lj}}-\tan ^{-1}\left(\mathrm{Q}_{\mathrm{j}} / \mathrm{P}_{\mathrm{j}}\right)\right)
$$

The constant current dynamical equations are derived as follows. For the original system, the bus current phasors are related to the the bus voltage phasors and the bus admittance matrix $Y$ through

$$
\left[\begin{array}{l}
I_{g} \\
I_{1}^{g}
\end{array}\right]=\left[\begin{array}{ll}
Y_{88} & Y_{g 1} \\
Y_{18} & Y_{11}
\end{array}\right]\left[\begin{array}{l}
V_{8} \\
V_{1}^{8}
\end{array}\right]
$$

where the subscript 1 denotes the load buses to be eliminated and the subscript $\mathrm{g}$ denotes the intemal generator buses that are 
preserved. After simple algebraic manipulations, the current-voltage relationship reduces to

where

$$
I_{g}=Y^{\propto q} V_{B}+D^{\propto q} I_{1}
$$

$$
\begin{aligned}
& Y^{\propto 1}=Y_{g g}-Y_{g 1} Y_{11}^{-1} Y_{l g} \\
& D^{\propto \mathcal{G}}=Y_{g 1} Y_{1 !}^{-1}
\end{aligned}
$$

The first term in the equation represents the contributions to generator current injections from generator voltages and the second term represents the load currents' contributions. Hence the swing equations for the internal generator busses are:

where

$$
\begin{gathered}
\delta_{\mathrm{i}}=\omega_{\mathrm{i}} \\
\mathrm{M}_{\mathrm{i}} \omega_{\mathrm{i}}=\mathrm{P}_{\mathrm{mi}}-\mathrm{D}_{\mathrm{i}} \omega_{\mathrm{i}}-\mathrm{P}_{\mathrm{ci}}
\end{gathered}
$$

$$
\begin{aligned}
P_{e i}= & V_{g i} \sum_{j_{g e n}} Y_{i j}^{e q} V_{g j} \cos \left(\delta_{j}-\delta_{j}-\phi_{i j}\right) \\
& +V_{g i} \sum_{j_{10 a d}} D_{i j}^{e q} I_{1 j} \cos \left(\delta_{i}-\varphi_{1 j}-\psi_{i}^{q q}\right)
\end{aligned}
$$

and $Y_{1}^{e q} \angle \phi f$ are the entries of $Y^{\circ q}$ and $D_{i}^{e q} \angle \psi_{i}^{e q}$ are the entries of Doq. This equation shows the dependence of the system dynamics on the values of the equivalent current injections $I_{1 j}<\varphi_{1 j}$. The equivalent currents depend in turn on $V$ and $\delta$. This explains why a loadflow must be run whenever it is necessary to update the equivalent current injections. The fast-decoupled power flow method is used for this purpose and its calculation time is included in the execution speeds given below. Fourth-order Runge-Kutta is used for each integration step within a given constant current model.

The algorithm for piecewise constant current integration is as follows. Define the base point be the point about which the most recent linearization occurred.

1. Choose the parameter $\gamma$ which specifies when to update the equivalent currents.

2. Integrate the constant current swing equation and check whether any of the generator angles have exceeded their base point values by $\gamma$. If so, then update the swing equations, otherwise do not update.

\section{Repeat Step 2 until the prediction interval is finished.}

This algorithm was tested on the New England 39 bus system with constant PQ loads. Several faults were simulated and their execution times and accuracies were measured. The computation time does in fact depend on the fault because the frequency of updates depends on the swing trajectory. It should also be noted that the coefficient matrices $Y \circ q$ and $D e q$ are assumed to be known before beginning the computation. Table II shows the range of solution times and generator angle error rates obtained by the piecewise constant current technique as compared with the exact constant $P Q$ solution method. The timing results were obtained for an HP $9000 / 720$ workstation which is several years out of date. An HP9000/735 model workstation which has just arrived and is waiting to be set up, is expected to run the programs three times faster.

Table II: Execution Speeds and Accuracies for Piecewise Constant Current Model

\begin{tabular}{lll} 
& CPU Time (sec.) & Error (deg.) \\
\cline { 2 - 3 }$y=5$ degrees & $.47-.59$ & $2.9-8.6$ \\
$\gamma=10$ degrees & $.41-.48$ & $6.0-17.8$
\end{tabular}

The parameter $\gamma$ controls the update frequency. Numbers shown are the execution time in seconds for integrating 10 machine angles one second into the future.

Table II indicates that between 5 and 10 degree updates, there is a large improvement in error and a small increase in CPU time in switching from 10 to 5 degrees. Simulations for the 39-bus system are therefore expected to run accurately and much faster than real-time on the HP9000/735.

\section{CONCLUSIONS}

The ability to obtain synchronized phasor measurements from around the system is expected to enable improved emergency response for maintaining system reliability. At the minimum, it seems that one should be able to predict with moderate accuracy what is going to happen in the near furure following a transient event. If one could predict what would happen under a variety of remedial control actions, then one could subsequently implement the best of those controls if the prediction is performed fast enough.

In the absence of an a priori known reduced-order model, the best one can do is extrapolate future behavior on the basis of past observations. We have developed a real-time clustering estimation - integration (CEI) algorithm to predict future behavior a short time into the fuare without relying on prior knowledge of the power system model. This is accomplished by fitting a very low order equivalent model to the dynamics of the particular event in progress, and solving the model forward in time to predict future behavior. Through systematic testing of this algorithm on the New England 39 bus system, we obtain reasonable success using a 2-machine equivalent for the CEI method, and show how the method could enhance the performance of a protection strategy against losses of synchronism such as the French Defence Plan. We highlight the importance of systematic testing by pointing out that 3 and 4 machine equivalent models prove adequate in a limited number of cases but have unacceptable performance overall. In doing so we also show that realistic precision phasor measurement data must be used in simulation in order to reach the proper conclusions about real world performance.

If a reduced-order model is available ahead of time, we show how its solution can be found in real-time. For a model as complex as the New England 39 bus system with composite loads, faster than real-time solution using post-fault phasor measurements as the initial condition can be performed using the piecewise constant current load approximation technique. For larger models a pattern recognition approach would be required. Previous publications have demonstrated the ability of a pattern recognition tool such as decision trees to predict future behavior based on a short sampling of the post-fault phasor measurements. A pattern recognition approach has greater flexibility in model complexity because the simulation of training set data is performed off-line and can be parallelized. Nevertheless, a fast and accurate simulation method such as the piecewise technique is needed for updating the decision tree logic in response to changing conditions. Hence the piecewise solution technique benefits both the real-time solution approach, and the off-line training of a pattern recognition tool.

\section{ACKNOWLEDGEMENTS}

This work was supported in part under the Electric Power Research Institute agreement RP3022-09. This work was also supported in part under subcontract \# 19X- SC203C for Energy Division Oak Ridge National Laboratory managed by Martin-Marietta Energy Systems Inc. for the US Department of Energy under contract \# DE-AC05-840R21400. Computer results were generated at the Comell National Supercomputer Facility which is funded in part by the National Science Foundation, New York State, and the IBM Corporation. 


\section{REFERENCES}

[1] A.G. Phadke, "Synchronized Phasor Measurements in Power Systems", IEEE Computer Applications in Power, Vol. 6, No. 2, pp. 10-15, 1993.

[2] A.G. Phadke et al., "Synchronized Sampling and Phasor Measurements for Relaying and Control". IEEE PES Winter Meeting, Columbus, Ohio, February 1993 (93 WM 039-8-PWRD).

[3] R.P. Schulz, L.S. VanSlyck, and S.H. Horowitz, "Applications of Fast Phasor Measurements on Utility Systems", PICA Proc., pp. 49-55, May 1989. [4] V. Centeno et al., "Adaptive Out-of-Step Relaying Computer Applications in Power, Vol. 6, No. 4, pp. 12-17, 1993.

[5] Ph. Denys et al., "Measurement of Voltage Phase for the French Future Defence Plan Against Losses of Synchronism", IEEE Trans. on PWRD, PWRD-7, No. 1 , pp. 62-69, 1992.

[6] C. Counan et al., "Major Incidents on the French Electric System: Potentiality and Curative Measures Studies", IEEE Trans. on PWRS, PWRS-8, No. 3, pp. 879-886, 1993.

[7] M. Bidet, of Electricité de France, in a personal communication subsequent to the presentation of "Contingencies System Against Losses of Synchronism Based on Phase Angle Measurements", at the IEEE PES 1993 Summer Meeting in a panel session on "Applications and Experience in Power System Monitoring with Phasor Measurements", 1993.

[8] T.L. Baldwin, L. Mili, and A.G. Phadke, "Dynamic Ward Equivalents for Transient Stability Analysis", IEEE PES 1993 Winter Meeting, Columbus, 1993.

[9] T.L. Baldwin, L. Mili, and A.G. Phadke, "Wand-type Equivalents for Transient Stability Analysis", Proceedings of the IFAC International Symposium on Control of Power Plants and Power Systems, Munich, Germany, March 9-11, 1992.

[10] S.M. Rovnyak S.E. Kretsinger, J.S. Thorp, and D.E. Brown, "Decision Trees for Real-Time Transient Stability Prediction", IEEE PES 1993 Summer Meeting, 93 SM 530-6 PWRS.

[11] M.H. Kent et al., "Dynamic Modeling of Loads in Stability Studies", IEEE Trans. on PAS, PAS-88, No. 5, pp. 756-763, May 1969.

[12] E. Vaahedi et al., "Load Models for Large-Scale Stability Studies from End-User Consumption", IEEE Trans. on PWRS, PWRS-2, No. 4, pp. 864-872, 1987.

[13] M.R. Brickell, "Simulation of Staged Tests in the Ontario Hydro Northwestem Region", PICA Proc., pp. 357-364, Cleveland, Ohio, 1979.

[14] J.C. Giri, "Coherency Reduction in the EPRI Stability Program", IEEE Trans. on PAS, PAS-102, No. 5, pp. 1285-1293, 1983.

[15] Y.-Y. Hsu and C.-H. Wu, "Experience with Dynamic Equivalencing of a Longitudinal Power System", Electrical Power and Energy Systems, vol. 13, no. 1 pp.2-8, 1991.

[16] M.A. Pai, Energy Function Analysis for Power System Stability, Kluwer, Boston, 1989.

[17] M. Szechtman et al., "DC Multiinfeed Study - Progress Report: Task 1: Development of an Appropriate System to be Studied", draft version of EPRI Research Project 2675 progress report (private communication). See also M. Szechtman et al., "DC Multiinfeed Study", EPRI Research Project 2675-5 Interim Report, April, 1992.

[18] Y. Xue, Th. Van Cutsem, and M. Ribbens-Pavella, "A Simple Direct Method for Fast Transient Stability Assessment of Large Power Systems", IEEE Trans. on PWRS, Vol. 3, No. 2, pp. 400-412, 1988.

[19] J.A. Hartigan, Clustering Algorithms, John Wiley \& Sons, New York, 1975.

[20] S.E. Kretsinger, S.M. Rovnyak, D.E. Brown, and J.S. Thorp, "Parallel Decision Trees for Predicting Groups of Unstable Generators from Synchronized Phasor Measurements", Precise Measurements in Power Systems Conference, Washington, D.C., 1993.

Steven M. Rovnyak received the B.S. in electrical engineering, the A.B. in mathematics, and the M.S. in electrical engineering from Comell University. Between 1986 and 1988 he spent summers and a fall term researching optical computing and neural networks at the BDM Corporation, McLean, VA. He is presently pursuing the $\mathrm{Ph} . \mathrm{D}$. in electrical engineering at Comell University. Mr. Rovnyak is a member of Phi Beta Kappa, Phi Kappa Phi, and a student member of the IEEE.

Chih-Wen Liu received the B.S. degree in Electrical Engineering from National Taiwan University, Taipei, Taiwan, in 1987. He received M.S. in 1992 and the $\mathrm{Ph} . \mathrm{D}$. in 1994 from Comell University in Electrical Engineering. His research interests include analysis of power system dynamics and applications of real-time phasor measurements to power system protection and control.

Jin Lu received the B.S. and M.S. degrees in electrical engineering from Nanjing Institute of Technology, Nanjing, China in 1982 and 1985 respectively. He received the Ph.D. in electrical engineering from Comell University in 1990. He is currently an electrical engineer at the ORA Corporation, a computer software firm. His research interests are in the areas of control and optimization of large scale systems with an emphasis on power systems.

Weimin $\mathrm{Ma}$ received his B.S. degree from Xi'an Jiatong University, Xi'an, China in 1982. He held a lecture position in the Systems Analysis Group of the School of Management Engineering at Xi'an Jiaotong University from 1982 to 1985 . He received the M.S. and Ph.D. degrees from Cornell University in 1987 and 1991, respectively. His interests include power systems, control and dynamic systems, and computational sciences.

James S. Thorp (S'58-M'63-SM'80-F 89) received the B.E.E, M.S., and Ph.D. degrees from Comell University, Ithaca, NY. He joined the faculty at Cornell in 1962 where he is currently a Professor of Electrical Engineering. In 1976 he was a Faculty Intern at the American Electric Power Service Corporation. He was an Associate Editor for IEEE TRANSACTIONS ON CIRCUITS AND SYSTEMS from 1985 to 1987 . In 1988 he was an Overseas Fellow at Churchill College, Cambridge, England. $\mathrm{He}$ is a member of the IEEE Power System Relaying Committee, CIGRE, Eta Kappa Nu, Tau Beta Pi, Sigma Xi. 


\section{Discussion}

Carson W. Taylor, Bonneville Power Administration, Portland Oregon: This is an interesting paper.

The authors seem to equate generator voltage angle with generator rotor angle. They also consider phasor measurements as state variables for system dynamics. Have the authors confirmed that generator terminal voltage angle is a good estimate of rotor angle? Have they considered synthesis of rotor angle from terminal measurements? In actual practice many phasor measurement transducers are located on the EHV or HV bus of generating plants. Considering a classical generator model, the impedance from the high side bus to the internal voltage is around $0.4-0.5$ per unit.

On page 2, the authors indicate that phasor measurements give better results than only local measurements [4]. Have the authors compared their method with advanced methods using local measurements such as the BPA $R-R$ dot controller $[\mathrm{A}, \mathrm{B}]$ ? In a real system, the $R-R$ dot controller operated without failure for a ten year period.

Regarding the statement "The first occurrence of 180 degree phase angle difference between PMU locations is the earliest that one could possibly initiate remedial control in the absence of prediction," conventional out-of-step relays and the $R-R$ dot controller trip "on-the-way-in" well before 180 degrees. This has been common practice in the western interconnection for several decades.

How do the authors justify constant PQ loads in transient stability simulation?

In the conclusions, the authors describe a decision tree approach using post-fault phasor measurements. Assuming the authors mean voltage angles [10], have the authors demonstrated that voltage angles (and their first and second derivatives) are better than other variables?

\section{References:}

A. J. M. Haner, T. D. Laughlin, and C. W. Taylor, "Experience with the R-Rdot Out-of-Step Relay," IEEE Transactions on Power Delivery, Vol. PWRD-1, No. 2, pp. 3539, April 1986.

B. C. W. Taylor, J. M. Haner, L. A. Hill, W. A. Mittelstadt, and R. L. Cresap, "A New Out-of-Step Relay with Rate of Change of Apparent Resistance Augmentation," IEEE Transactions on Power Apparatus and Systems, Vol. PAS-102, No. 3, pp. 631-639, March 1983.

Manuscript received August 19, 1994.

S. Rovnyak, C.-W. Liu, J. Lu, W. Ma, J. Thorp. Mr. Taylor raises some very important issues as far as this research is concerned, and in order to respond to his points, the different parts of our work are briefly summarized as follows:

Classifier technique for predicting future angle stability from a short window of post-fault generator voltage angle measurements $[10,20, \mathrm{C}]$.

Clustering-Estimation-Integration (CEI) described in this paper.

Piecewise Constant Current load model approximation for power system simulation described in this paper and in [D].
The discusser correctly observes that we have used the phrase "generator rotor angles" interchangably with "generator voltage angles" and "phasor measurements". What we are intending is that when you have a phasor measurement unit (PMU) located on the EHV or HV bus of a generating plant, that you will have a suitably detailed model of the generator for simulation purposes, but also have a classical generator model consisting of a voltage source behind a transient reactance. The internal voltage in the classical model is easily computed from the terminal voltage and current phasor measurements:

$$
E_{\text {internal }}=E_{\text {terminal }}-I_{\text {terminal }} * X_{d}^{\prime},
$$

where $X^{\prime}{ }_{d}$ is the transient reactance. In the more general situation where phasor measurements are not taken directly from generator buses, the reduced $Y_{\text {bus }}$ matrix relating the measured voltages $V_{m}$ and the generator (internal) voltages and currents $V_{g}, I_{g}$ can be used solve for the generator (internal) voltages $v_{g}[E]$. From

$$
\left[\begin{array}{l}
I_{g} \\
0
\end{array}\right]=\left[\begin{array}{ll}
Y_{11} & Y_{12} \\
Y_{21} & Y_{22}
\end{array}\right]\left[\begin{array}{l}
V_{g} \\
V_{m}
\end{array}\right] \text {, }
$$

the second set of equations

$$
\mathrm{Y}_{21} \mathrm{~V}_{\mathrm{g}}+\mathrm{Y}_{22} \mathrm{~V}_{\mathrm{m}}=0
$$

can then be solved for the generator voltages by least squares.

Since our research thus far has used classical generator models in simulation and has used the generator internal voltage angles from the state equations as the simulated phasor measurements, the next step in sophistication would be to use detailed generator models for large scale simulation while taking phase angle measurements from the generator terminal voltages and currents, and converting these back into generator internal voltage angles for the classical model. One could then test the ability to predict future behavior using internal voltage angles computed from the phasor measurements, and this would seem to be a natural extension to more detailed power system models.

If one were using a pattern recognition approach as described in the earlier publications, a classifier could likely be trained to associate generator terminal phasor measurements directly with the prediction of future behavior. It would need to be tested whether a classifier would perform better using generator terminal phasor measurements as classifier inputs, or else using the computed internal voltage angles of the associated classical models. The issue is also raised in the discussion on using variables other than voltage angles as input variables for the classifier, and this is something that we have not tested. Decision trees do have an advantage of being able to select the profitable variables from an overinclusive set of potential input variables. It is likely that including other variables such as line power flows would bring an improvement in decision tree performance without an overly large increase in the amount of computation required for training.

The discusser correctly points out that the R-Rdot relay has been successfully employed in the western United States to implement a controlled separation of the western North American interconnection into two islands, and asks for a comparative justification of our own methods. Unfortunately we do not have comparative simulation results but we can at least make the following observations. The reference [4] about adaptive ont-of-step relaying between Florida and Georgia using synchronized phasor measurements describes the design and installation of this PMU based protection system but does not report on testing and operation to the extent and detail contained in $[A, B]$. It therefore remains an open question whether phasor measurements can ultimately give better results than using only local measurements as the $R-R$ dot relay does.

While the $\mathrm{R}-\mathrm{Rdot}$ technique is a direct competitor of the 
CEI method, or more properly stated the other way around, the pattern recognition tools $[10,20, \mathrm{C}]$ have some potential superiorities to both. For example the simulation testing of decision trees and neural networks showed them capable of predicting loss of synchronism within the next four seconds; which far exceeds the rather minimal predictive abilities of the CEI and R-Rdot methods. As for a comparison between CEI and R-Rdot, it is our understanding that the R-Rdot installation in the western United States was motivated by a desire to refine the decision boundary in out-of-step relays to more closely approximate the actual stability boundary instead of being set over-conservatively. It was the same objective which motivated the development of CEI, and the simulations in this paper show the number of false trips to be very small ( $\left.{ }^{-} 2 \%\right)$ using hundreds of marginally stable transient stability runs.

With regard to the R-Rdot relay tripping "on the way in" well before 180 degrees, we should at least point out that we were using the criterion of whether any two of the voltage angles were 180 degrees apart, which occurs slightly before the electrical centers become 180 degrees apart. In fact it is the latter quantity that we require to be predicted to exceed 180 degrees before declaring a transient event in progress to be unstable. This is the reason for the CEI method being accurate and late to trip. To the extent that the R-Rdot controller is able to trip on an unstable swing well before 180 degrees we would answer the discusser's comment by saying that the R-Rdot relay is performing prediction as much or possibly somewhat more so than the CEI. Note that the CEI could trip earlier if the criterion of 180 degree phase angle difference were lowered, at the expense of increasing the number of false trips on stable swings. Unless the R-Rdot decision boundary exactly separates the stable swings from the unstable swings, there is going to be some conservatism in the relay operation because mistakes in the category of false trips can be tolerated.

The $R-R$ dot relay in $[A, B]$ was designed through simulation and testing to disconnect the western US interconnection into two pre-determined islands whenever loss of synchronism is imminent. By contrast we have shown in simulation that the CEI method is able to predict with some accuracy the divergence in angle of any group of generators from the rest. Although we have only tested our method on a small system (10 generators) assuming phasor measurements from all the generators, we have done so with the hope that $\mathrm{CEI}$, as well as pattern recognition, could be generalized to larger systems by taking measurements from a representative subset of generators or generator equivalents. If successful this extension of the CEI method to large scale systems would offer an advantage over the present $\mathrm{R}-\mathrm{Rdot}$ controller in being able to prescribe different separation patterns for different disturbances and outages. We do however recognize that additional work must be performed to make sure the system will be separated into "islandable" areas.
Finally in response to the question on constant $P Q$ load models, we should clarify that our simulations of the CEI and pattern recognition methods used constant impedance loads and classical generator models because they were easiest to simulate. As indicated earlier these methods can and should be extended to more complex power system models with non-linear loads, detailed generator models and so forth. The piecewise constant current approximation technique in Section 4 was therefore offered for the purpose of simulating power system models with constant $P Q$ and other voltage dependent loads.

Although we have not ourselves investigated the justification of constant PQ loads in transient stability simulation, we can point to the simplified equivalent of the western North American interconnection developed mostly in CEPEL, Brazil under EPRI project RP 2675-4 17,F]. There seem to be two main versions of this simplified equivalent according to $[\mathrm{F}]$ :
(I)
121 buses and 29 generators
(II) 133 buses and 41 generators

where (II) is an expanded version of (I). A variant of (I) for which we have obtained data has 173 buses and 29 generators because intermediate buses were added for the purpose of representing series compensated lines in a format where the capacitor elements are represented separately from the transmission line elements $[\mathrm{F}]$. Another version [G] reportedly has 131 buses and 29 generators, while [17] reports "approximately 130 busses" and 40 generators. In any case, both [17] and the data for the 173 bus version have fractions of the load in the South California and PG\&E areas represented as constant PQ loads. Since the simplified equivalent in [17] was designed to replicate on a smaller scale the dynamic behavior of the full 2158 bus network, we suggest that this justifies the occasional need for constant $P Q$ loads in transient stability simulation.

\section{References}

[C] S.M. Rovnyak, Algorithms for Real-Time Transient Stability Prediction in Electric Power Systems, Ph.D. Thesis, Cornell University, Ithaca, NY, 1994.

[D] C.-W. Liu, Adaptive Out-of-Step Reloying Algorithms Based on Synchronized Phasor Measurements: Real-Time Transient Stability Prediction and Transiently Chaotic Swing Detection, Ph.D. Thesis, Cornell University, Ithaca, NY, 1994.

[E] J.S. Thorp et al., "Some Applications of Phasor Measurements to Adaptive Protection", IEEE Trans. on PWRS, Vol. 3, No. 2, pp. 791-798, 1988.

[F] C.A. Canizares, Voltage Collapse and Transient Energy Function Analyses of $A C / D C$ Systems, Ph.D. Thesis, University of Wisconsin, Madison, WI, 1991.

[G] M. Szechtman et al., "The Behavior of Several HVDC Links Terminating in the Same Load Area", Cigre Paper 14-201, August, Paris, 1992.

Manuscript received October 28, 1994. 